\title{
BMJ Open Research protocol for platelets in out-of- hospital cardiac arrest: an observational, case-controlled, feasibility study to assess coagulation and platelet function abnormalities with ROTEM following out-of-hospital cardiac arrest (PoHCAR)
}

To cite: Skorko A, Thomas M, Mumford A, et al. Research protocol for platelets in outof-hospital cardiac arrest: an observational, case-controlled, feasibility study to assess coagulation and platelet function abnormalities with ROTEM following out-of-hospital cardiac arrest (PoHCAR). BMJ Open 2017;7:e015663. doi:10.1136/ bmjopen-2016-015663

- Prepublication history for this paper is available online. To view these files please visit the journal online (http://dx.doi org/10.1136/bmjopen-2016015663)

Received 3 January 2017 Revised 19 May 2017 Accepted 2 June 2017

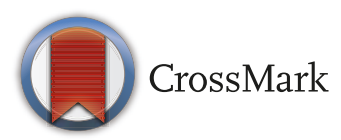

For numbered affiliations see end of article.

Correspondence to

Dr Agnieszka Skorko; a.skorko@ nhs.net

\section{ABSTRACT}

Introduction 0ut-of-hospital cardiac arrest (OHCA) has an annual incidence of approximately 60000 in the UK. Less than $10 \%$ of those who receive resuscitation survive to hospital discharge. For OHCA of a presumed cardiac cause, the optimal antiplatelet therapy is currently unknown. Previous studies indicate that a procoagulopathic state exists postcardiac arrest which may contribute to the formation of thrombi and contribute to poor outcomes. However, the administration of antiplatelet therapies needs to be balanced against the increased risk of bleeding that these individuals face.

Methods and analysis This observational feasibility study will recruit 30 individuals who achieve return of spontaneous circulation post-OHCA, are admitted to a single tertiary centre over a 6-month period and meet Utstein cohort criteria (witnessed cardiac arrest, VF or pulseless VT and cardiac cause of arrest likely). Rotational thromboelastometry and platelet function assessment will be performed on hospital arrival, postemergency percutaneous coronary intervention (PCI) and 12 hours, 24 hours and 48 hours post-PCl. As a comparator, 30 individuals presenting to our institution with ST-segment elevation myocardial infarction and undergoing primary $\mathrm{PCl}$ will have the same blood sampling performed. Plasma samples will be retained and batch tested on completion of the study for levels of protein $\mathrm{C}$, protein $\mathrm{S}$, thrombin-antithrombin complex, thrombin, antithrombin, plasminogen activator inhibitor-1, plasmin-antiplasmin complex, d-dimer, platelet factor-4, P selectin, E selectin and prothrombin fragments 1 and 2. 30-day follow-up for complications will be undertaken.

Ethics and dissemination This study has been approved by the Wales REC 7Research Ethics Committee. The results will be submitted to peerreviewed medical journals and suitable national and international meetings. Results will be locally disseminated via our patient and public interest group.

\section{Strengths and limitations of this study}

The research site is a busy regional cardiac arrest and primary percutaneous coronary intervention centre, serving a population of 1000000 .

- The study is supported by a multidisciplinary steering committee, with a strong track record of successfully delivering clinical trials in the relevant fields of emergency medicine, cardiology and platelet assessment.

- The rotational thromboelastometry system is used currently as a research tool within the host institution, supported by experience in a wide range of platelet function techniques.

- This is an observational feasibility study with a relatively small sample size.

- Cardiac arrest is the end point of a number of pathological processes. Although the Utstein cohort are being selected to reduce heterogeneity, enrolled patients are likely to have different causes of cardiac arrest.

- No literature exists as to the size of the effect we are expecting with regard to platelet function over time; therefore, we have not been able to complete a power calculation.

- Volume of nasogastric aspirate measurement offers only indirect evidence of drug absorption.

Trial registration number Pre-results; ISRCTN34122839.

\section{INTRODUCTION}

It is estimated that 60000 out-of-hospital cardiac arrests (OHCAs) occur in the UK each year, of which 31000 have resuscitation commenced or continued by emergency medical services. ${ }^{1}$ Data from Ambulance Quality Indicator statistics demonstrate that the survival rate postcardiac arrest to hospital 
discharge is around $8 \%$, with regional variability within the UK from $6.7 \%$ to $9.4 \% .{ }^{23}$ Due to the heterogeneous nature of OHCAs and difficulties in standardising data for publication, a uniform model was proposed by an international task force in 1991. The 'Utstein comparator cohort' is an internationally agreed OHCA cohort comprising those who have OHCA of presumed cardiac origin, have a witnessed OHCA and ventricular fibrillation or ventricular tachycardia as the initial heart rhythm. ${ }^{4}$ Within the UK in the period 2014-2015, this standardised subset of OHCAs achieved return of spontaneous circulation (ROSC) in $49 \%$ of instances and the survival to hospital discharge was $26.3 \%$. $^{2}$ The Utstein group forms a small proportion of OHCA admissions, but are more representative of cardiogenic OHCA.

Cardiogenic OHCA results from myocardial ischaemia/infarction secondary to coronary artery occlusion, the extreme end of the acute coronary syndrome spectrum. The mainstay of treatment following OHCA due to coronary occlusion is twofold: rapid restoration of blood flow to the myocardium and instigation of neuroprotective mechanisms to minimise hypoxic brain injury.

Throughout the UK a network of regional centres offers emergency percutaneous coronary intervention (PCI) and coronary stent insertion 24 hours a day to expedite restoration of blood flow. Following stent insertion, it is of utmost importance to ensure vessel patency and avoid the complication of in-stent thrombosis. To this end, administration of antiplatelet and antithrombotic therapies to suppress thrombus formation is vital.

In the context of OHCA, there is evidence to suggest that a prothrombotic tendency exists. ${ }^{6}$ Activation of thrombosis may help explain poorer outcomes, so the administration of antiplatelet drugs is potentially even more vital in this subset of patients.

However, a delicate balance exists between the risk of coronary thrombosis and the bleeding risk posed by potent platelet inhibitors, particularly in the context of a critically ill individual. Aspirin has been the traditional mainstay of therapy in these patients, but significant advances in the inhibition of platelet activity have occurred in recent years. Clopidogrel, a second-generation thienopyridine has been superseded by the third-generation drugs prasugrel and ticagrelor. These newer drugs are given in conjunction with aspirin after coronary stenting in a regimen known as dual antiplatelet therapy (DAPT).

Platelet inhibition by any of these oral drugs takes a period of time to become effective. In the acute phase, until these drugs can begin to exert an effect, the administration of intravenous platelet inhibitors, such as a glycoprotein IIb/IIIa receptor antagonist (abciximab, tirofiban or eptifibatide), direct thrombin inhibitors (bivalirudin) or intravenous ADP receptor antagonists (cangrelor) to prevent immediate thrombosis of stents is common during PCI.

Despite rapid PCI and DAPT, the vast majority of patients presenting after cardiac arrest will remain comatose, so they will be admitted to an intensive care unit
(ICU) for postresuscitation care. Until recently, a critical component of postcardiac arrest care was the neuroprotective strategy of therapeutic hypothermia (TH). ${ }^{7}$ This treatment involves cooling of the individual to a core body temperature of $32^{\circ} \mathrm{C}-34^{\circ} \mathrm{C}$ for $24-48$ hours, followed by a subsequent gradual rewarming period. Since the publication of the 2013 paper by Nielsen et al the role of TH has become less clear and has been superseded by targeted temperature management (TTM) where temperature is controlled in the range of $33^{\circ} \mathrm{C}-36^{\circ} \mathrm{C}$ for $48-72$ hours ${ }^{8}$. However, at present, equipoise exists as to whether TH or TTM is the superior neuroprotective strategy, so clinicians currently make an assessment of appropriateness on a case-by-case basis. Profound hypothermia has been shown to impair platelet activation and induce fibrinolysis, thus increasing the risk of bleeding. This becomes more profound at progressively lower temperatures.

Current understanding of the relative effectiveness of all antiplatelet drugs following OHCA and particularly in therapeutically cooled humans is limited. Evidence suggests that these patients have a greater rate of in-stent thrombosis, with rates of $10 \%$ reported. ${ }^{9}$ There are several recognised factors that specifically attenuate the effectiveness of DAPT in this population such as delayed administration, poor absorption, altered metabolism, multiorgan failure and the effect of TTM which may all impact on the efficacy of antiplatelet drugs.

In addition to the iatrogenic interventions that alter coagulation and platelet function, data suggest that a coagulopathy develops rapidly after cardiac arrest. ${ }^{6}$ Postcardiac arrest syndrome is a recognised proinflammatory state with upregulation of mediators such as TNF $\alpha$ and interleukin-6, both of which are known to cause platelet activation and thrombin production. ${ }^{10}$ This ultimately leads to a prothrombotic state and may explain why even after restoration of coronary flow outcomes remain poor post-OHCA.

In order to assess coagulation and platelet function dynamically, a number of techniques are available. One of the best established is rotational thrombolestometry (ROTEM). This system uses the change in blood's viscoelastic properties during clot formation to infer the balance of coagulation, platelet function and fibrinolytic activity and displays the results in a graphical form. Its ability to map clot formation kinetics makes it an effective clinical tool for near patient assessment of dynamic coagulation. Using a variety of reagents, the system also makes it possible to appreciate the differential activities of the tissue factor (or extrinsic), contact activation (or intrinsic) and fibrin pathways of the coagulation system.

No single, ideal method for assessing dynamic platelet function currently exists. We will use a novel platelet module in conjunction with the ROTEM machine to assess P2Y12 and thrombin receptor function and so understand intrinsic platelet activity and subsequent impact of DAPT therapies. This new module uses the principle of impedance aggregometry but has the advantage of being integrated into the ROTEM system and 
is more automated than other methods. We will undertake an observational case-controlled feasibility study to describe the coagulopathy of cardiac arrest and how it changes over the first 48 hours following PCI, DAPT and TTM administration in Utstein comparator individuals admitted to a single UK tertiary cardiac arrest centre following OHCA. A second, control group will recruit those who are admitted to the same institution for emergency primary PCI following acute myocardial infarction (STEMI, ST-segment elevation myocardial infarction).

\section{METHODS AND ANALYSIS \\ Primary study aim}

The primary aim of this study is to assess and describe platelet function and coagulation at predefined time points, in Utstein cohort patients admitted to hospital with ROSC following OHCA.

An initial assessment of coagulation on admission will aim to identify what derangements of coagulation and platelet function occur postcardiac arrest prior to any pharmacological intervention. Subsequent changes in clotting and platelet function following PCI, administration of antiplatelet therapy, and during the participant's ICU stay (with particular focus on the effect of temperature control strategies) will be described.

\section{Secondary study aims}

This feasibility study will be used to establish a sound methodology for assessing coagulation and platelet function in participants admitted to our institution with ROSC following OHCA. The results will be used to generate research hypotheses regarding the optimal strategy for antiplatelet therapy in Utstein criteria postOHCA participants for assessment in future clinical trials. We will collect data to compare the rates of bleeding, blood transfusion and in-stent thrombosis within 30 days of admission between the OHCA and STEMI groups.

\section{Study population}

All adult patients following OHCA who meet Utstein cohort criteria presenting to the Bristol Royal Infirmary in the 6-month period from September 2016 to February 2017 will be eligible for enrolment into this study.

The 'Utstein comparator cohort' is an internationally agreed OHCA cohort comprising those who have OHCA of presumed cardiac origin, have a witnessed arrest and ventricular fibrillation or ventricular tachycardia as the initial heart rhythm.

Individuals who are pregnant will not be eligible for enrolment as platelet function is known to alter during pregnancy. Due to the ethical implications, individuals who are known to have dementia, or are detained under either the Mental Health Act or by Her Majesty's Prison Service will not be eligible. Finally, individuals who it is deemed will not benefit from ICU admission will not be enrolled.

As a control group, individuals who present to the Bristol Royal Infirmary coronary catheter laboratory (CCL) for primary PCI as treatment for STEMI will be eligible. Individuals will not be eligible if they have experienced a period of cardiac arrest prior to admission, do not meet the ESC criteria for STEMI, are not offered primary or are undergoing rescue PCI. This group forms the most suitable control group as they experience the same underlying pathological process of coronary plaque rupture and occlusion and receive the same initial DAPT, but without subsequent ICU interventions.

\section{Study design}

All OHCA and STEMI individuals will be screened at admission to our institution. Following confirmation of enrolment and verbal assent in the STEMI arm, two citrated blood samples will be taken immediately. These samples will be processed within 2 hours on the ROTEM delta and platelet modules. The remaining blood sample will be centrifuged and the plasma stored at $-80^{\circ} \mathrm{C}$.

Further blood sampling for ROTEM analysis will occur at the end of PCI and at 12 hours, 24 hours and 48 hours post-PCI (figure 1). On each occasion, two citrate samples will be taken and processed as described above. Due to the 24 hours nature of OHCA admissions, achieving blood sampling at all time points may prove challenging. Therefore, priority will be given to collecting samples at

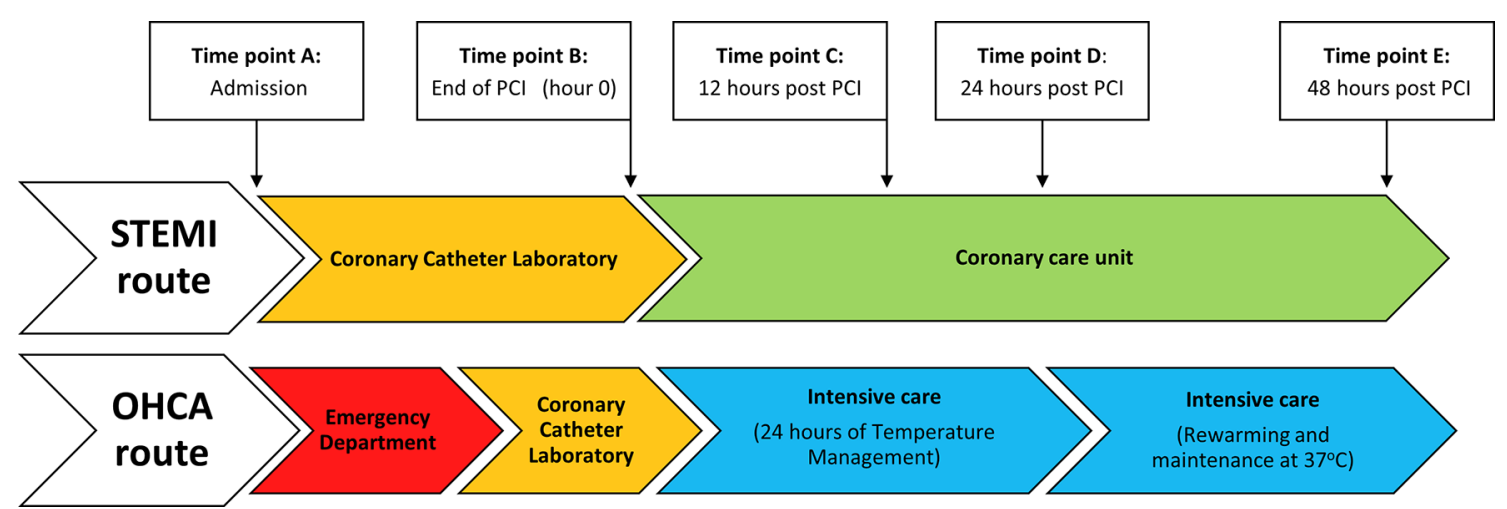

Figure 1 Timeline of blood sampling in each study arm. OHCA, out-of-hospital cardiac arrest; PCI, percutaneous coronary intervention; STEMI, STE elevation myocardial infarction. 
time points 1, 4 and 5 in OHCA survivors and 1 and 4 in the STEMI cohort.

Basic demographic data will be collected about participants in each study arm, including past medical and medication histories. Information will be recorded about the cardiac arrest itself and arrival time in ED. Events in the CCL will be documented (therapy undertaken, number and site of stents and any complications) as will the doses and timing of antiplatelet and anticoagulant therapies administered. During the subsequent 48 hours the following data will be recorded: arterial blood gas results, haemoglobin, haematocrit, platelet count, international normalised ratio (INR), activated partial thromboplastin time (APTT), creatinine, use of renal replacement therapy, 24 hourly fluid balance, body temperature and target temperature, antiplatelet therapies given with timings recorded and any deviation from prescription. Doses of vasopressors and sedation will be recorded as will daily APACHE II and SOFA scores. Any instances of bleeding, blood product transfusion, return to CCL, or vomiting will be recorded on the standardised case record form (CRF). Volume of nasogastric aspirate will be documented as an indirect indicator of drug absorption in OHCA participants.

Complications occurring within the first 30 days (including but not limited to: bleeding, need for transfusion, return to CCL for in-stent thrombosis or further PCI), length of ICU, coronary care unit (CCU) and total hospital stay as well as 30-day survival will all be recorded. Bleeding severity will be quantified using the Bleeding Academic Research Consortium scale. This scale as has been advocated by several authors for assessment of bleeding following cardiac events. ${ }^{11}$ Classification of post-PCI complications will follow the Academic Research Consortium recommended guidelines. ${ }^{12}$

Given the feasibility nature of this study, a screening $\log$ will be kept to identify the individuals who were not enrolled and the reasons for this.

\section{Outcome measures}

The primary outcome measure will be a description of ROTEM coagulation and platelet parameters following OHCA in the Utstein comparator cohort, from admission to 48 hours post-PCI. Coagulation and platelet function will be correlated with the data gathered to generate hypotheses regarding baseline coagulation and platelet function in OHCA participants and to what extent these are influenced by the medications administered and core body temperature. The same analysis will be undertaken in the control group of participants undergoing treatment for STEMI who we anticipate receive comparable DAPT therapy without the subsequent ICU interventions.

\section{Data analysis plan}

Data will be collected from medical notes and entered onto a CRF before being inputted into a predesigned, password protected excel spreadsheet. A 10\% sample of CRFs will be reviewed by another researcher against medical notes to ensure data validity and accuracy of data entry.

Data will be analysed on an intention-to-treat basis to examine; choice of DAPT administered, timing of administration (pre-PCI, at PCI, post-PCI), preadmission exposure to antiplatelet therapy, degree of absorption of drug (as inferred from NG aspirates) and temperature management strategy in the OHCA group.

To track the platelet function and investigate its association with events such as DAPT administration and TTM, graphs will be used in the first instance. The results of platelet function and coagulation assessment will be plotted against time for all participants with different coloured markers for those in the two cohorts, for those before and after the administration of anticoagulants and those who have experienced bleeding events. This graphical representation will allow an understanding of the data, its limitations and its strengths. Natural logs of platelet function will be used rather than raw platelet function if the graphs show that there is more variability with high values than low values (skewed data).

A formal linear regression of platelet function will be carried with a robust error structure to allow for the multiple sampling points per participant. The robust error structure allows the calculation of SEs by dividing the SD by the root of the number of participants rather than the number of data points, so it does not over inflate the statistical significance for data which are not independent. This regression will allow us to quantify the difference in clotting risk for participants with and without cooling or participants with and without DAPT while controlling for the time the samples were taken.

Due to the small number of participants in this data set and the fact that it is not powered to detect differences the $p$ values themselves will not be reported, but the CI around the effect sizes will be reported to allow for the planning of full definitive studies.

To establish the methodology for any future definitive work, the feasibility parameters for various methodological issues will be described using 95\% CIs. We will report the data needed to plan a future trial such as the percentage of eligible participants recruited to the trial, the percentage which failed to achieve adequate consent, the percentage of blood samples which did not yield useful results and the percentage of participants who had samples taken within 2 hours of the predefined time points.

To characterise the coagulation and platelet function in OHCA and STEMI participants we will tabulate the laboratory results for each of these groups and separate the results by time for those in both of the cohorts. We will also tabulate the data separately for those receiving and not receiving DAPT at each time point. A further tabulation will be made comparing the coagulation and platelet function in each group by type of DAPT delivered (clopidogrel vs prasugrel vs ticagrelor). In each case, we will report mean, median, $\mathrm{SD}$ and range. 
Given the non-interventional nature of this study a formal data management committee has not be formed. Instead the study trial committee will meet on a monthly basis during patient recruitment to review the recruitment and safety reporting processes.

\section{ETHICS AND DISSEMINATION}

The study has been granted and will be carried out in accordance with Health Research Authority approval, encompassing Research Ethics Committee (REC) and local research and development approvals.

The enrolment of participants after cardiac arrest is ethically challenging and requires special consideration as regards consent. Conducting research in emergency situations where a patient lacks capacity is regulated by the Mental Capacity Act (2005) for England and Wales. The occurrence of a cardiac arrest out of hospital is unpredictable. Within seconds of cardiac arrest, a person becomes unconscious and thus incapacitated. As a result, it is impossible to obtain prospective consent from the individual. In this setting, it is also impractical to consult next of kin or an independent registered medical practitioner without placing the potential participant at risk of harm from delaying treatment. Therefore, it is not practical to seek any form of consent before initial enrolment. Instead a model of deferred consent will be used when enrolling participants.

Consent will be sought from participants by an appropriately trained member of the research team to continue follow-up data collection as soon as the individual is able to give informed consent. However post-OHCA care necessitates the participant to be sedated for at least 72 hours, and therefore, the time until they regain capacity is greatly deferred.

Given this situation, initial advice about ongoing inclusion in the study will be sought from a personal consultee (as identified by the participant's clinical team). This consultee will be informed of the participant's enrolment in the study and their view sought as to whether the participant would have been willing to be involved, under the provisions of the Mental Capacity Act. The research team will liaise with clinical staff to determine the optimal time to make this approach, ideally within 24-96hours of ICU admission. A full explanation of the purpose of the study, the protocol, interventions thus far and future interventions will be given. An information sheet will be provided. It will also be explained that once the participant regains capacity, they will be asked to give informed consent to continue in the study. Should the participant not wish to be involved their decision will override that given by the consultee.

If this personal consultee does not feel the participant would have agreed to enrolment, they will be withdrawn from the study. In this instance, additional advice will be sought as to whether the data and blood samples already collected may be retained or whether these should be destroyed.
If the personal consultee feels the participant would have agreed to enrolment, a written opinion will be obtained from this consultee and the participant will continue in the study.

In instances where no personal consultees are identified, a professional consultee will be sought from the lead clinician involved in the participant's care. This individual will be independent of the research team and be in no way associated with the study. It is envisaged that this approach will be made within 24-96 hours of admission to ICU.

Participants themselves will be approached for consent once they have recovered sufficiently from the OHCA and regained capacity, as judged by the clinical team responsible for that participant's care. If it is felt the participant does not have capacity to give informed consent every effort will still be made to explain the study to the individual, in the presence of their personal consultee and an information leaflet left. This leaflet will include contact details for the research team should the individual have questions in the future.

If the participant does have capacity and does not consent, they will be withdrawn from the study, regardless of the earlier opinion of their personal consultee.

Finally, if no assent or consent can be obtained (eg, the participant dies and no appropriate consultee can be identified), then the participant will be withdrawn from the study and all data and blood samples relating to that individual will be destroyed.

Participants presenting with STEMI will be consented by a member of the research team as soon as clinically appropriate after arrival in the CCL prior to PCI. An assent model will be used whereby the individual is given a brief verbal description of the study and asked to state a preference. No formal capacity assessment is performed. If verbal assent is given, an independent witness is asked to sign a form. Once the participant has completed their acute treatment and had an opportunity to recuperate the research team will approach the patient again. During this visit, the study will be explained in detail, a patient information leaflet will be provided and written consent sought.

If an individual or consultee initially agrees to participation but later withdraws they will be asked to indicate whether they are willing for the information and samples collected thus far to be retained or for all these to be destroyed in a secure manner.

It will be made clear at the time of consent or assent that withdrawal can occur up to the point that the information and samples become anonymised. Anonymisation will occur on the day of study completion; 30 days after the enrolment of the final participant. After this time, it is impossible to trace which samples or results belong to whom.

On completion of the study, data will be analysed as per the data analysis plan. Results will be submitted to appropriate peer-reviewed journals for publication and to national and international conferences. The results 
will be presented to the local patient and public interest group who assisted with refining the protocol, along with staff at our institution. It is anticipated that anonymous da125443125443ta will ultimately be deposited in an appropriate data repository. Despite anonymisation of the results, the potentially sensitive nature of the data means that it will be stored under an appropriately managed access process rather than a fully 'open data' arrangement and access restricted to researchers who have signed a data sharing agreement and gained appropriate ethics committee approvals. The final decision regarding data access will be made in conjunction with the publishing journal.

\section{Author affiliations}

${ }^{1}$ Department of Anaesthesia, University Hospitals Bristol NHS Foundation Trust, Bristol Royal Infirmary, Bristol, UK

${ }^{2}$ Department of Haematology, University Hospitals Bristol NHS Foundation Trust, Bristol, UK

${ }^{3}$ Department of Cardiology, University Hospitals Bristol NHS Foundation Trust, Bristol, UK

${ }^{4}$ Research and Innovation Office, University Hospitals Bristol NHS Foundation Trust, Bristol, UK

${ }^{5}$ Department of Emergency Care, University Hospitals Bristol NHS Foundation Trust, Bristol, UK

Contributors AS, MT, RG and JB prepared the manuscript. TJ, AM and EG assisted with the study design and protocol.

Funding This work is supported by the Bristol CardioVascular Biomedical Research unit. Dr Skorko is funded by a National Institute for Health Research Integrated Academic Training (NIHR ACF) award in Intensive Care Medicine. Grants from the Resuscitation Council (UK) and David Telling Charitable Trust will assist with serum sample processing.

Competing interests None declared.

Ethics approval Wales REC 7 research ethics committee.

Provenance and peer review Not commissioned; externally peer reviewed.

Data sharing statement It is anticipated that anonymous data will ultimately be deposited in an appropriate data repository. Despite anonymisation of the results, the potentially sensitive nature of the data means that it will be stored under an appropriately managed access process rather than a fully 'open data' arrangement and access restricted to researchers who have signed a data sharing agreement and gained appropriate ethics committee approvals. The final decision regarding data access will be made in conjunction with the publishing journal.

Open Access This is an Open Access article distributed in accordance with the Creative Commons Attribution Non Commercial (CC BY-NC 4.0) license, which permits others to distribute, remix, adapt, build upon this work non-commercially, and license their derivative works on different terms, provided the original work is properly cited and the use is non-commercial. See: http://creativecommons.org/ licenses/by-nc/4.0/

(c) Article author(s) (or their employer(s) unless otherwise stated in the text of the article) 2017. All rights reserved. No commercial use is permitted unless otherwise expressly granted.

\section{REFERENCES}

1. Perkins GD, Cooke MW. Variability in cardiac arrest survival: the NHS Ambulance Service Quality Indicators. Emerg Med J 2012;29:3-5.

2. NHS England. Ambulance Quality Indicators (AQI) January 2016. 2016.

3. Perkins GD, Brace-McDonnell SJ, OHCAO Project Group. The UK Out of Hospital Cardiac Arrest Outcome (OHCAO) project. BMJ Open 2015;5:e008736.

4. Cummins R, Chamberlain D, Abramson N, et al. Recommended guidelines for uniform reporting of data from out-of-hospital cardiac arrest: the 'Utstein style'. Resuscitation 1991;22:1-26.

5. Hazinski M, Nolan J, Aickin R, et al. International Consensus on Cardiopulmonary Resuscitation and Emergency Cardiovascular Care Science with treatment recommendations. Circulation 2015;2015:S2-39.

6. Adrie C, Monchi M, Laurent I, et al. Coagulopathy after successful cardiopulmonary resuscitation following cardiac arrest: implication of the protein $\mathrm{C}$ anticoagulant pathway. J Am Coll Cardiol 2005;46:21-8.

7. Peberdy MA, Callaway CW, Neumar RW, et al. Part 9: post-cardiac arrest care: 2010 American Heart Association Guidelines for Cardiopulmonary Resuscitation and Emergency Cardiovascular Care. Circulation 2010;122(18 Suppl 3):S768-S786.

8. Nielsen N, Wetterslev J, Cronberg T, et al. Targeted temperature management at $33^{\circ} \mathrm{C}$ versus $36^{\circ} \mathrm{C}$ after cardiac arrest. N Engl J Med 2013;369:2197-206.

9. Joffre J, Varenne O, Bougouin W, et al. Stent thrombosis: an increased adverse event after angioplasty following resuscitated cardiac arrest. Resuscitation 2014;85:769-73.

10. Bro-Jeppesen J, Kjaergaard J, Wanscher M, et al. The inflammatory response after out-of-hospital cardiac arrest is not modified by targeted temperature management at $33^{\circ} \mathrm{C}$ or $36{ }^{\circ} \mathrm{C}$. Resuscitation 2014;85:1480-7.

11. Mehran R, Rao SV, Bhatt DL, et al. Standardized bleeding definitions for Cardiovascular clinical trials. Circulation 2011;123:2736-47.

12. Cutlip DE, Windecker $S$, Mehran R, et al. Clinical end points in coronary stent trials: a case for standardized definitions. Circulation 2007; 115:2344-51. 